serum-induced thrombosis, for if the serum is injected into the portal vein thrombotic activity is much more transient, while ligating the portal vein prolongs thrombotic activity (Wessler et al., 1967). Tindall (1966) has shown that oral contraceptives reduce the rate of elimination of bromsulphalein into the bile while apparently increasing hepatic blood flow, and diethylstilboestrol alone is now known to have a similar effect (Clinch and Tindall, 1968). The increased levels of factor IX in puerperal women receiving diethylstilboestrol may be due to the effect of this drug on liver function.

The effect of the administration of diethylstilboestrol on this single coagulation factor is reported for three reasons. First, though we are investigating changes in fibrinogen and other coagulation factors in these patients, the changes in factor IX levels are already statistically significant at 0.001 level. Secondly, factor IX is of interest in relation to thromboembolism, and our findings may be of relevance in view of the increased incidence of this disease in women receiving oral contraceptives (Medical Research Council, 1967), in puerperal women receiving diethylstilboestrol (Daniel et al., 1967), and in men treated with oestrogens for coronary thrombosis (Oliver and Boyd, 1961). Finally, the findings also suggest a possible new approach to the management of factor IX deficiency (Christmas disease).

\section{Summary}

Factor IX levels in plasma have been estimated in late pregnancy and in the puerperium. Puerperal administration of diethylstilboestrol was accompanied by significantly higher factor IX levels than were found in late pregnancy or in the puerperium in women who were lactating or in whom lactation had been suppressed without the use of drugs. This difference was most pronounced in women of low parities. The finding is discussed in relation to the observation that factors IX, XI, and XII influence venous thrombosis in vivo, and also in relation to the reported association between oestrogen administration and thromboembolism.

Our thanks are due to Mr. J. G. Lawson, consultant obstetrician, United Cardiff Hospitals, for permission to include his patients in this study.

\section{REFERENCES}

Clinch, J. A. D., and Tindall, V. R. (1968). Proc. Brit. pharmacol. Soc.

In press. Davidson, E., and Tomlin, S. (1963). 7. clin. Path., 16, 112.

Fresh, J. W., Ferguson, J. H., and Lewis, J. H. (1956). Obstet. and Gynec., 7,117

Hardisty, R. M., and Ingram, G. I. C. (1964). Bleeding Disorders : Investigation and Management. Oxford.

Kasper, C. K., Hoag, M. S., Aggeler, P. M., and Stone, S. (1964). Obstet. and Gynec., 24, 242.

Mrdicai Research Council (1967). Brit. med. 7., 2, 355.

Nilsson, I. M., and Kullander, S. (1967a). Acta obstet. gynec. scand., 46, 273.

Nilsson, I.M., and Kullander, S. (1967b). Acta obstet. gynec. scand., 46 , 286

Vossel, H. L. Lanzkowsky, P., Levy, S., Mibashan, R. S., and Hansen, J. D. L. (1966). Thrombos. Diathes. haemorrh. (Stuttg.), 16, 185 Oliver, M. F., and Boyd, G. S. (1961). Lancet, 2, 499.

Ratnoff, O. D., and Holland, T. R. (1959). Ann. N.Y. Acad. Sci., 75, 626.

Ross, R. A. (1963). Amer. 7. Obstet. Gynec., 86, 77.

Rutherford, R. N., Hougie, C., Banks, A. L., and Coburn, W. A. (1964) Obstet. and Gynec., 24, 886 .

Tindall, V. R. (1966). Blair Bell Lecture, Royal College of Obstetricians and Gynaecologists.

Todd, M. E., Thompson, J. H., Bowie, E. J. W., and Owen, C. A. (1965). Mayo Clin. Proc., 40, 370.

Wessler, S., and Reimer, S. M. (1960). 7. clin. Invest., 39, 262.

Wessler, S., Yin, E. T., Gaston, L. W., and Nicol, I. (1967). Thrombos. Diathes. haemorrh. (Stuttg.), 18, 12.

\title{
Presentation of Midgut Malrotation in Adults
}

\section{H. BRENDAN DEVLIN,* M.A., M.D., M.CH., F.R.C.S., F.R.C.S.I. ; R. S. MAURICE WILLIAMS, $†$ B.A., M.B., B.CHIR.}

\author{
J. W. PIERCE, $\ddagger$ M.D., M.R.C.P., D.M.R.D.
}

\section{Brit. med. F., 1968, 1, 803-807}

Though midgut malrotation is a common cause of intestinal obstruction in the newborn, scant attention is given to this developmental anomaly as a cause of symptoms in adults. The standard textbooks describe the features of this neonatal obstruction, and then mention that the condition may first present as a great rarity in later life.

In the past 18 months five adults with recurrent symptoms due to midgut malrotation have presented to one surgical firm in St. Thomas's Hospital. These cases have had many features in common, so much so that when the fifth patient attended the outpatient department the correct diagnosis was made on the history and clinical features alone.

Each patient gave a story of recurrent attacks of abdominal colic since childhood. These were often severe and lasted up to 72 hours ; then the pain settled spontaneously. In two cases the end of the attack was sometimes accompanied by a bout of blood-stained diarrhoea. Each case had been variously diagnosed and treated previously; in two of them barium studies of the stomach and duodenum only had failed to reveal the true condition.

- Senior Surgical Registrar, St. Thomas's Hospital, London S.E.1.

† House Surgeon, St. Thomas's Hospital, London, S.E.1.

¥ Consultant Radiologist, St. Thomas's Hospital, London S.E.1.
These cases are described below, and the clinical features and radiographic and operative findings are discussed.

\section{Case 1}

A man aged 20 was admitted to hospital as an emergency case in February 1966. He gave a history of severe abdominal colic and vomiting for 72 hours. He had had similar attacks of central abdominal colic frequently since early childhood. Each attack would last about 48 hours and then settle spontaneously. No satisfactory diagnosis had been made, and on one occasion he had been disciplined for malingering when an attack coincided with the end of a period of leave from the Services. A previous barium-meal examination was reported as showing "a normal stomach and duodenum."

On admission to St. Thomas's he had generalized abdominal tenderness, an empty rectum, and all the signs of dehydration due to his vomiting. An emergency laparotomy showed a midgut volvulus, and at its apex a persistent vitello-intestinal duct connected the mass of intestines to the umbilicus. An enteric cyst and a urachal remnant were also present at the umbilicus. After these abnormal ducts and cysts had been divided from the small gut a complete midgut malrotation was found, with the small intestine lying in the right abdomen. The midgut arose from a single long 
aarrow mesentery. An area of gangrenous small intestine was resected and the abdomen was then closed.

\section{Case 2}

A man aged 22 presented in February 1967 with a history of bouts of severe upper abdominal colic and diarrhoea since the age of 3 months. These attacks occurred at intervals of six to eight weeks. Examination in the outpatient department disclosed some tenderness in the left side of the abdomen, and a tentative diagnosis of Crohn's disease was made. A routine barium-meal examination was reported as normal, but a formal small-bowel meal showed a complete failure of intestinal rotation, with the small intestines occupying the right side of the abdomen and the colon the left side (Figs. 1 and 2). A diagnosis of recurrent small intestinal volvulus was made.

At laparotomy the whole bowel from the duodenum to the descending colon was found to be suspended from a single narrow dorsal mesentery based on the origin of the superior mesenteric vessels. The duodenum ran caudally in a straight line from its first part onwards. The caecum lay in the left side of the abdomen and the ileum entered it from the right (Fig. 2). The mesentery along the axis of the superior mesenteric artery was sutured to the posterior abdominal wall in order to prevent further volvulus (see Fig. 9). There has been no recurrence of symptoms since the operation.

\section{Case 3}

A married woman aged 54 was admitted in October 1962 with a history of episodes of central abdominal colic every two to three months since childhood. The attacks of pain were accompanied by vomiting, and then, after two to three days, as the pain was settling, she would have a bout of diarrhoea. At the age of 24 she had had an appendicectomy for "one of these attacks." Physical examination revealed some tenderness in the right lower abdomen, and a clinical diagnosis of Crohn's disease was made.

A barium small-bowel meal showed an unrotated duodenum with the jejunum lying in the right abdomen. The ileum entered the caecum from the right, and a barium enema confirmed that the

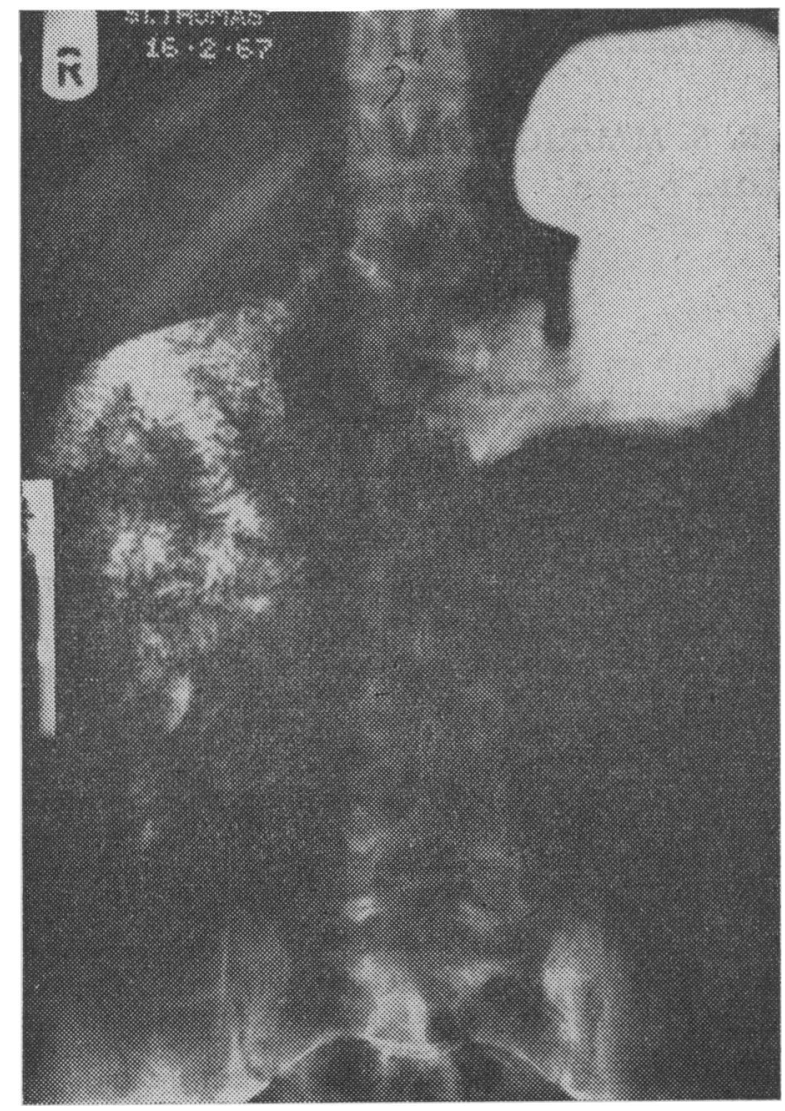

Fig. 1.-Case 2. Small-bowel meal showing the unrotated duodenum and the jejunum in the right abdomen. colon lay on the left and that there was a complete non-rotation of the midgut. In the small gut there were apparently areas of dilatation and narrowing which suggested Crohn's disease.

At laparotomy the small bowel was found to have a long mesentery which was very narrow at its origin. The duodenum was unrotated and twisted back on itself, and the ileum entered the

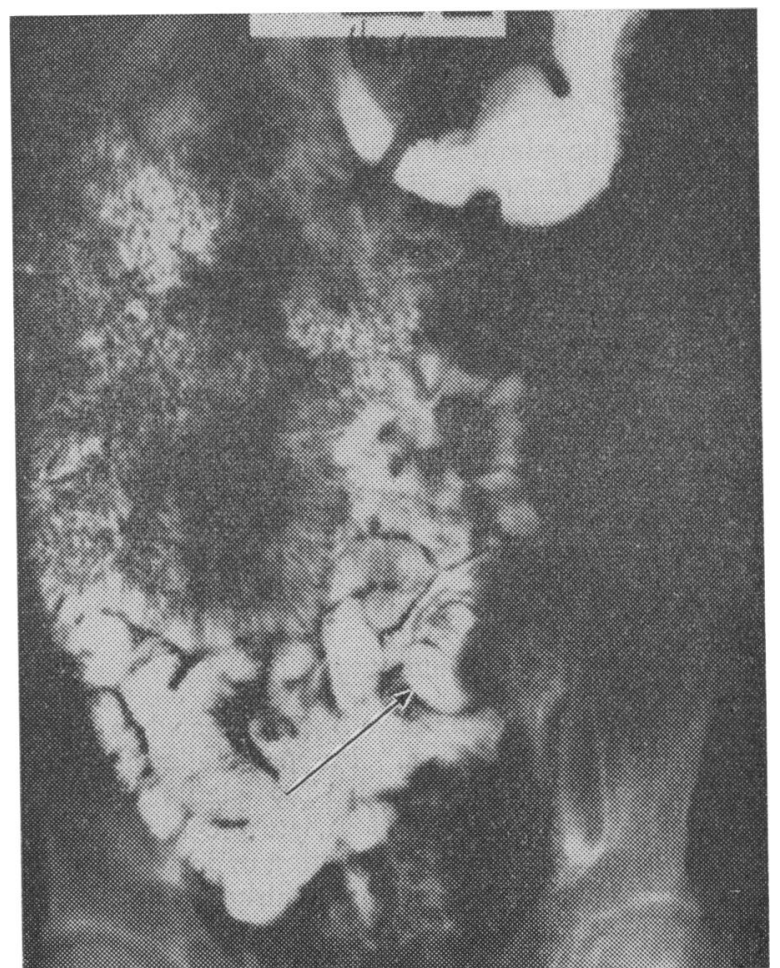

FIG. 2.-Case 2. Small-bowel meal after $1 \frac{1}{4}$ hours. This shows the small intestine in the right abdomen. The terminal ileum opens into the caecum, which lies to the left of the midline (arrow). Subsequent films in the series confirmed the midposition of the caecum and showed the colon in the left abdomen.

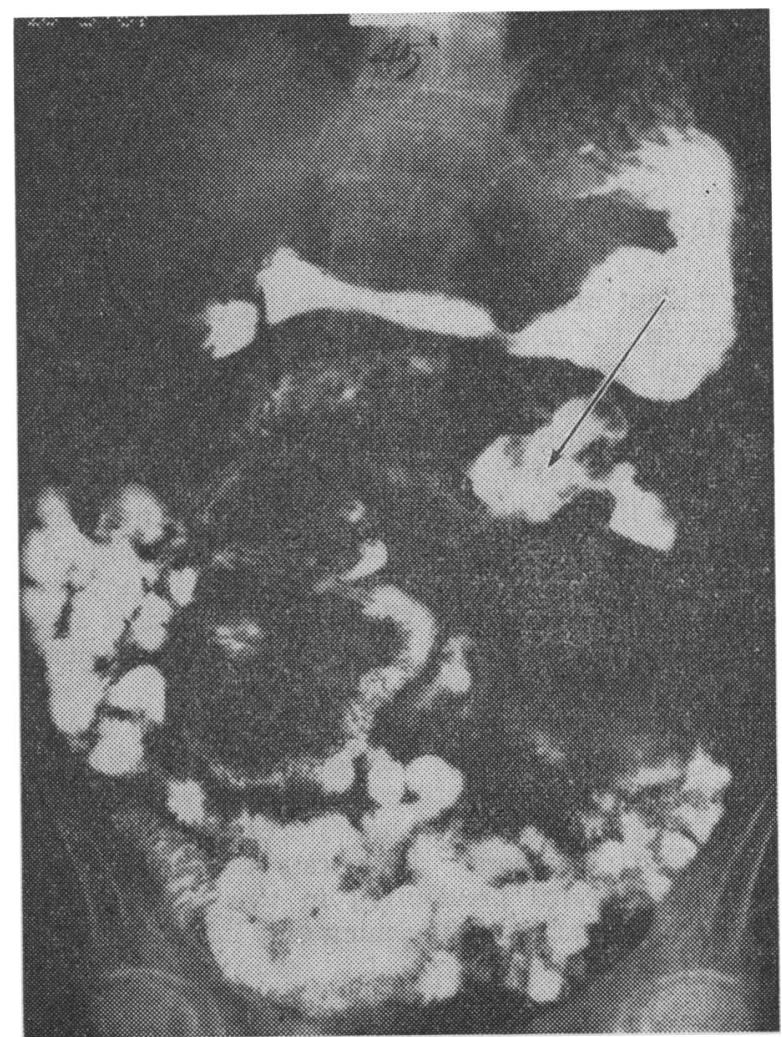

Fig, 3-Case 3 Barium small-bowel meal showing midgut malrotation and the caecum in the left upper abdomen (arrow). 
caecum from the right. No lesions of Crohn's disease were found. In order to prevent further episodes of midgut volvulus the posterior surface of the common mesentery was sutured to the posterior abdominal wall in the sagittal plane as far as the pelvic brim.

After this operation the patient was symptom-free for four years until December 1966, when she had another attack of colic and vomiting. She was seen for routine follow-up in April 1967, and in view of the recurrent symptoms the possibility of Crohn's disease was raised again. Barium studies at this time confirmed the midgut malrotation but did not entirely exclude Crohn's disease (Fig. 3). For this reason laparotomy was advised.

At operation the bowel was found to be densely adherent to the posterior abdominal wall ; there were no lesions of Crohn's disease, and no cause for the obstructive episode in 1966 could be demonstrated. Since operation there has been no return of the symptoms.

\section{Case 4}

A man aged 21 first attended in May 1967 with a history of bouts of central abdominal colic, vomiting, and diarrhoea since early childhood. These attacks occurred every three to four months ; they began suddenly and ended gradually, the end of the attack being heralded by the onset of diarrhoea, the stools sometimes being blood-stained. At the age of 8 he had had bilateral undescended testicles treated surgically. He had also been treated by a psychiatrist for "reactive depression" because of his undiagnosable abdominal pain. Physical examination was unrewarding, and on the basis of the history a tentative diagnosis of Crohn's disease was made.

Barium studies showed a midgut malrotation, with the small gut in the right abdomen and the large gut on the left (Figs. 4 and 5).

The patient was admitted for laparotomy in September 1967. At operation all the intestine from the duodenum to the descending colon was found to be suspended on a single dorsal mesentery. This mesentery arose from a narrow base high up in the abdomen and reached down to the pelvic brim. There were numerous areas of fine shiny fibrosis on the small bowel corresponding to sites of previous constriction when a midgut volvulus occurred. This primitive mesentery was sutured to the posterior abdominal wall in the sagittal plane along the axis of the superior mesenteric vessels.

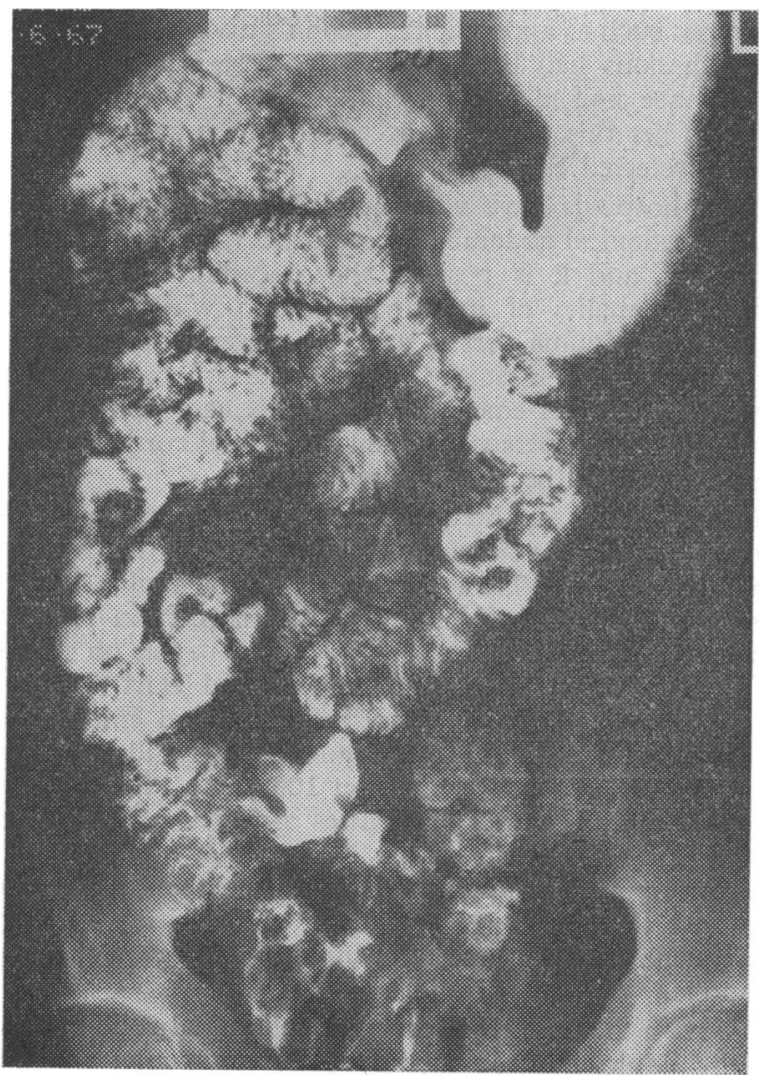

HIG. 4.-Case 4. Small-bowel meal.
He was also found to have a midline gall bladder and biliary apparatus, and the aorta bifurcated high at the level of the renal arteries.

\section{Case 5}

A girl aged 18 came to the outpatient department in July 1967 with a history of intermittent attacks of central abdominal colic, sometimes accompanied by vomiting, since the age of 2 . Each attack lasted up to 24 hours, beginning suddenly and settling gradually. These attacks occurred every few months and on occasions diarrhoea occurred towards the end of the attack.

On examination no abnormality was found in the abdomen. An initial diagnosis of midgut malrotation and intermittent volvulus was made. Barium studies of the small bowel confirmed this diagnosis (Fig. 6). In childhood this patient had been seen by a long succession of doctors, who had concluded that her pains were of psychogenic origin, and she was given psychiatric treatment. In 1963 a normal appendix had been removed at another hospital after an attack of her usual symptoms. Her general practitioner had at this time diagnosed "intermittent volvulus," but this diagnosis was ignored by the surgeon to whom she was referred.

At laparotomy in September 1967 it was found that though the caecum lay to the right of the midline, the small bowel, caecum, and ascending colon arose from a long mesentery with a narrow base. The duodenum and caecum were connected by a broad fibrous band (Ladd's band) and the duodenum was only partially rotated. Ladd's band accounted for the peculiar distribution of the small intestine into two compartments (Fig. 6). Ladd's band was excised and the mesentery and caecum were anchored to the posterior abdominal wall. A small follicular ovarian cyst was also excised.

The postoperative course has been uneventful, and to date the patient has had no recurrence of symptoms.

\section{Discussion}

Between the fifth and tenth weeks of intrauterine life the midgut develops within the umbilical sac. While it is outside the true abdominal cavity the bowel forms a loop which begins to rotate on the axis of the superior mesenteric vessels. This

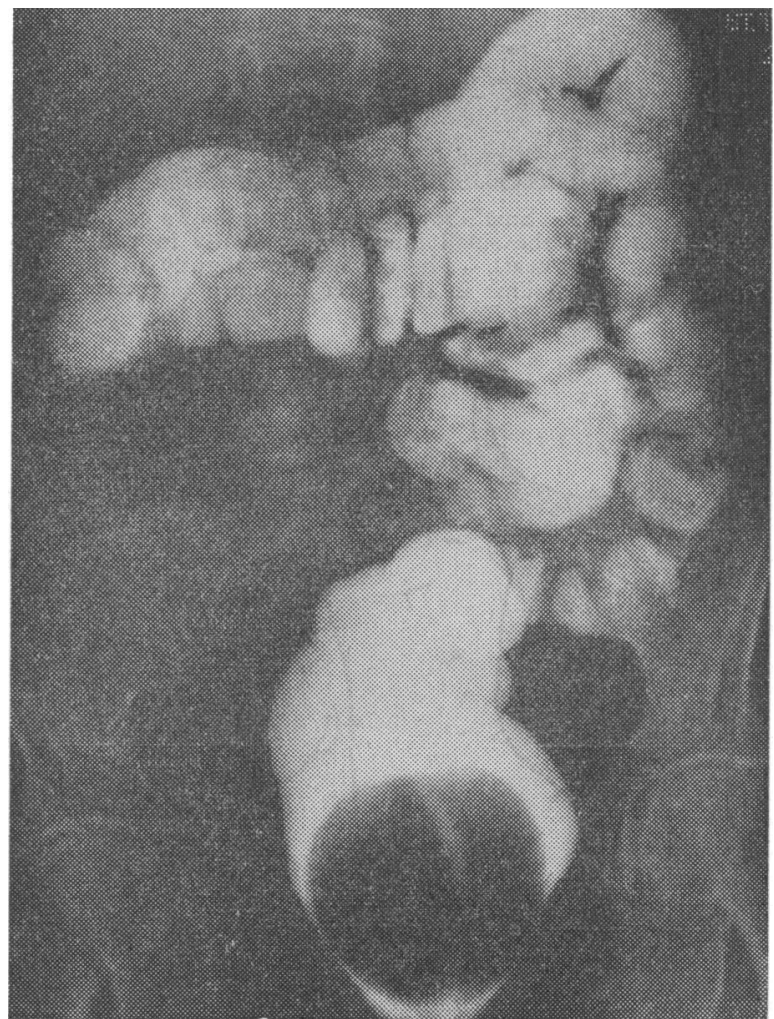

Fig. 5.- Ciase 4. Barium enema showing all the colon lying in the left abdomen. No intestinal rotation has taken place 
rotation is in an anticlockwise direction, and when it is eventually completed the gut has rotated through an arc of 270 degrees, so that the caecum and terminal ileum lie to the right and the colon lies fanned out around the periphery of the abdominal cavity. Subsequent to this the mesocolon becomes adherent to the posterior abdominal wall in the flanks and the intestines assume their adult disposition (Fig. 7).

Derangements of this process may occur at any stage; the gut may remain entirely unrotated and be suspended by a single mesentery as in Cases 1, 2, 3, and 4 (Fig. 8), or partial rotation may have taken place as in Case 5. Other developmental anomalies of the gastrointestinal system may complicate the picture, as in Cases 1, 4, and 5.

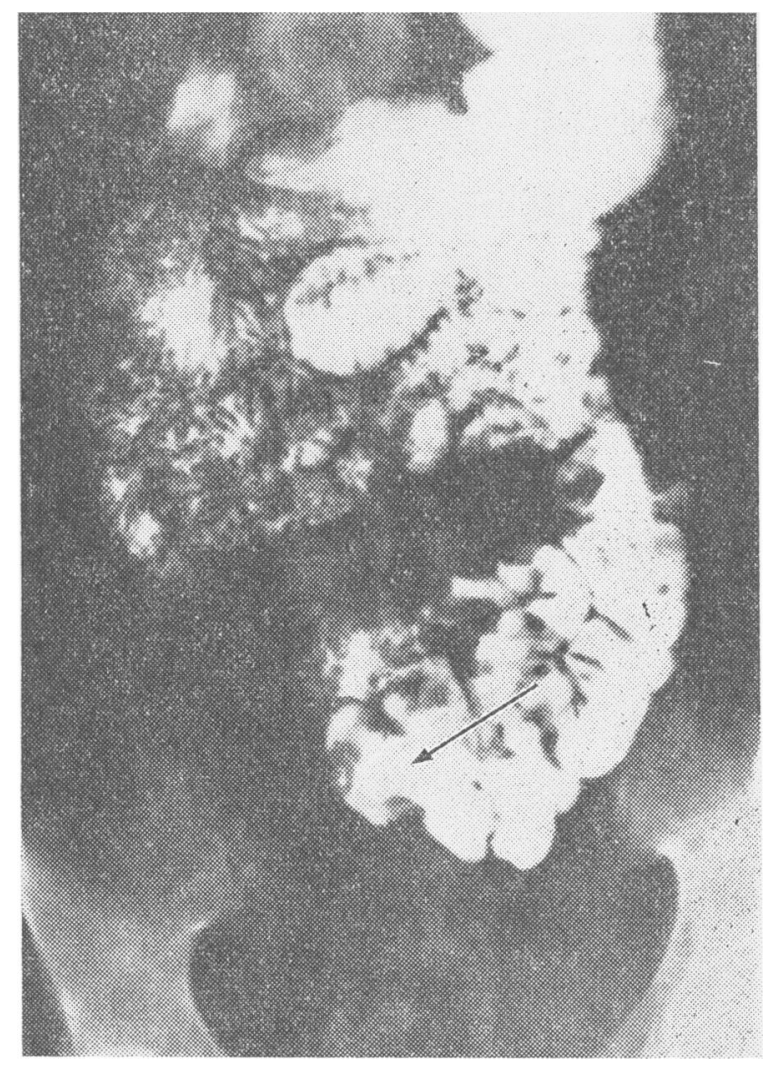

FIg. 6.-Case 5. Small-bowel meal. This shows an unrotated duodenum with the small intestine apparently in two "compartments" (see text). The caecum lies slightly to the right of the midline in the lower abdomen (arrow).

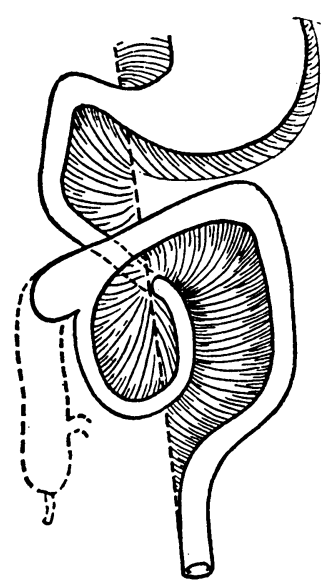

Fig. 7

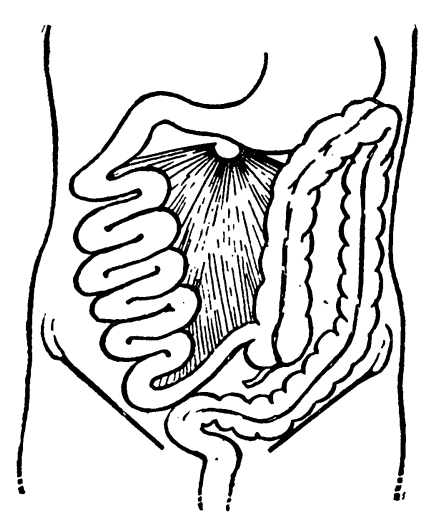

FIG. 8
FIG. 7.-Simplified diagram of the normal anticlockwise rotation of the midgut in the embryo. Fig. 8.-Diagram showing the distribution of the intestines when complete non-rotation is present. The narrow mesenteric origin is indicated.
Malrotation of the gut may be discovered accidentally at operation or may present with inflammation of a misplaced organ. It may also present with intestinal obstruction. In the newborn this condition is almost always accompanied by high intestinal obstruction due to compression of the duodenum. Snyder and Chaffin (1954) reported on 40 cases seen at the Los Angeles Children's Hospital. All 40 had high intestinal obstruction due either to adhesive bands (Ladd's band) running from the duodenum to the high caecum or to involvement of the duodenum in the base of a midgut volvulus. In the present series midgut volvulus occurred in four cases, and a combination of volvulus and band obstruction in the fifth.

The cases recorded here have many features in common. The patients were all apparently healthy adults who since childhood had had intermittent attacks of central abdominal colic unrelated to any precipitating cause. The attacks occurred at intervals of a few weeks to some months; sometimes they were accompanied by vomiting, and in all cases they often ended in a bout of diarrhoea, which in two cases was occasionally blood-stained. The pain started acutely but tended to fade out after 24 to 72 hours. Abdominal distension was not a significant feature of the attacks. Clinical examination between the attacks of ten showed no abnormality or at most minimal abdominal tenderness.

All these patients had long been permitted to suffer these periodically incapacitating symptoms without any definite diagnosis being made. The probable explanation for this was the absence of physical signs by the time the patient came to be examined by the doctor and the negative results yielded by routine investigations, such as cholecystography and pyelography. Even a conventional barium-meal examination did not suggest the diagnosis in some cases, presumably because after the first part of the duodenum had been screened further studies were not made of the transit of the contrast medium through the small gut. Overall the most likely reason for not making the diagnosis is simply because the clinical features did not correspond to any condition likely to be considered. One patient, a naval rating, had been disciplined for malingering, two had been referred to psychiatrists, and in the others diagnoses such as appendicitis, Crohn's disease, "spastic colon," and adhesions had been made. In Case 5 a shrewd general practitioner had diagnosed "intermittent volvulus" but had failed to get this diagnosis accepted by the surgeons to whom he had referred the case.

Most hospitals have a residue of patients with bizarre and of ten paradoxical abdominal disturbances, such as colic without distension, and it is possible that a certain number of these possess anomalies of intestinal rotation which predispose to temporary volvulus that is never severe enough to bring them to emergency laparotomy. Only one of our patients was operated on as an emergency. In this case non-rotation was combined with a persistent vitello-intestinal cord around which the volvulus had become strangulated.

In a search of the literature we found several papers dealing with malrotation as a cause of obstruction in the newborn and some isolated reports of its occurrence in adults, but we did not find any paper which discussed malrotation as a clinical problem in adults. The definitive clinical papers on this subject are those of Dott (1923) and Gardner and Hart (1934). Dott was concerned to classify these anomalies and emphasize them as a cause of neonatal obstruction. Gardner and Hart reviewed the literature and commented on 105 cases. Ladd and Gross (1941) described a series of 44 cases of which 26 were diagnosed in the first three weeks of life. The result of these and other paediatric papers has been perhaps to give the mistaken impression that this condition forcibly manifests itself in early life, consequently leading one to exclude it as a cause of symptoms in adults.

We wish to stress that recurrent abdominal colic in otherwise healthy adults may be caused by developmental anomalies of the gut, and though these derangements are extremely rare they 
should always be excluded before the patient is labelled as a psychiatric problem. The syndrome of bouts of colic and vomiting with little if any distension and diarrhoea to complete the episode is very characteristic of malrotation. The probable mechanism is a midgut volvulus with partial duodenal obstruction being relieved spontaneously. If some impairment of blood supply of the gut occurs, blood-stained diarrhoea may signify the culmination of the attack.

Investigations are of ten negative ; in particular barium-meal studies without full screening of the small gut will often fail

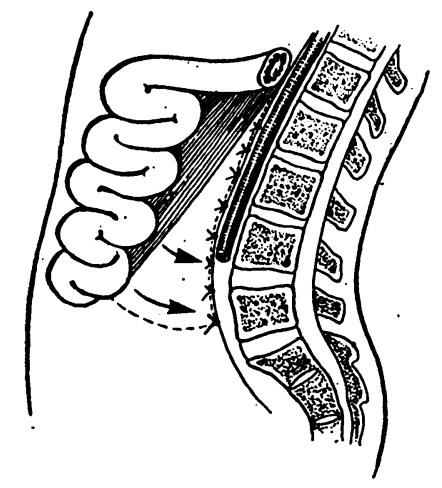

FIG. 9.-Diagrammatic saggital section of the peritoneal cavity illustrating the long narrow mesentery arising in the upper abdomen in midgut malsutured to the posterion this is peritoneum (dotted lines).

to make the diagnosis, and a misleading report, such as "stomach and duodenum normal," may further confuse the situation.

All these cases should have a laparotomy to confirm the diagnosis and to exclude other more dangerous causes of the symptoms. If intestinal malrotation is found any abnormal bands should be excised and an attempt made to suture the intestines to the posterior abdominal wall to prevent further volvulus (Figs. 8 and 9). When doing this it is important to incise the posterior parietal peritoneum and the posterior mesenteric peritoneal layer, and then to suture these peritoneal layers together. Unless the peritoneum is incised and sutured in this way adequate adherence of the mesentery to the parietes cannot be guaranteed. If the case is operated on urgently and strangulated bowel needs resecting, it is probably wise to defer this mesenteric suturing until a later date.

\section{Summary}

Five adult patients presented with a history of recurrent intestinal obstruction since childhood. Each obstructive episode lasted between 24 and 72 hours and was characterized by colic and vomiting, and diarrhoea towards the culmination of the attack. Physical examination between the attacks showed nothing of note. Barium studies of the whole intestinal tract revealed varying degrees of midgut malrotation, and laparotomy confirmed the diagnosis. At operation the mesentery was sutured to the posterior abdominal wall to prevent further episodes of volvulus. Symptomatic improvement followed this manœuvre. The pathology, investigation, and treatment of these cases are discussed.

We wish to thank Dr. B. Creamer, Mr. R. W. Nevin, and Mr. H. E. Lockhart-Mummery for permission to investigate and report on cases under their care.

REFERENCES

Dott, N. M. (1923). Brit. F. Surg., 11, 251.

Gardner, C. E., and Hart, D. (1934). Arch. Surg., 29, 942

Ladd, W. E., and Gross, R. E. (1941). Abdominal Surgery of Infancy and Childhood. Philadelphia.

Snyder, W. H., and Chaffin, L. (1954). Ann. Surg., 140, 368.

\title{
Dermatoses in Lobster Fishermen
}

\author{
W. E. BEER,* M.B., M.R.C.P.ED. ; MARGARET JONES, $\dagger$ B.SC. ; W. EIFION JONES, $\dagger$ B.SC., PH.D.
}

Brit. med. F., 1968, 1, 807-809

This study began as a result of our being consulted by a lobster fisherman (Case 1) for the elucidation of his problem of intense itching of the hands during the lobster-fishing season. $\mathrm{He}$ informed us that others in his locality, similarly engaged, had also complained of itching. Arrangements were therefore made, in the summer of 1967, to examine the other six men who, like this patient, operated in the Bardsey sound area at the tip of the Lleyn Peninsula in North Wales. There are some 21 other lobster fishers elsewhere on the north and south coasts of the Lleyn Peninsula, but these were not investigated.

The lobster-fishing season begins in May and lasts until October or November. The state of the tides and daylight govern the start and conclusion of the work each day. The method of fishing is to lay baited traps ("pots") on the seabed. These pots are weighted and attached to marker floats (see Fig.). The fishermen usually work singly, and the number of pots which each individual can look after depends on the time he can spend fishing; most of them have other occupations which take up the rest of their working time.

The bait used in the pots is composed of discarded fish from the Conway trawlers, which is bought in bulk and then salted by the lobster fishermen and stored for daily use. The hands of the fishermen are thus continually in contact with high concentrations of salt, which may in itself have an adverse effect on the skin.

The pots are hauled up daily, and though the men wear rubber boots and aprons they cannot avoid being soaked by water from the ropes. The lobsters caught have their claws secured by rubber bands and at the end of the day are transferred to a large pot kept out at sea and emptied once a week. During the winter, time is spent making new lobster pots and repairing and repainting boats.

It has been noticed that a growth of "slimy weed" becomes established on the ropes during the summer. This first appears at the start of the season and dies away in late July. It is limited to the upper portion of the rope only, where light conditions are presumably optimal for growth.

The possibility of our patient being sensitive to the "slimy weed" growing on the ropes was considered. This mass, though largely composed of colonial diatoms, in fact contained much more than merely "weed." Small animals and bacteria were also present in considerable quantities.

\footnotetext{
* Consultant Dermatologist, Caernarvonshire and Anglesey General Hospital, Bangor, Caernarvonshire.

† Marine Science Laboratories, Menai Bridge, Anglesey.
} 